\title{
Propuesta didáctica basada en múltiples formas de representación semiótica de los objetos matemáticos para desarrollar el proceso de enseñanza aprendizaje del cálculo diferencial. ${ }^{1}$
}

\author{
Ana Mercedes Báez ${ }^{2}$, Olga Lidia Pérez González ${ }^{3}$, Bartolo Triana Hernández ${ }^{4}$
}

Recibido, Febrero 07 de 2017

Evaluado, Marzo 12 de 2017

Aceptado, Marzo 24 de 2017

\author{
Referencia: Mercedes, A.; Pérez, O.; Triana, B. (2017). \\ "Propuesta didáctica basada en múltiples formas de \\ representación semiótica de los objetos matemáticos para \\ desarrollar el proceso de enseñanza aprendizaje del cálculo \\ diferencial". Revista Academia y Virtualidad, 10, (2), 20-30
}

\section{Resumen}

En el artículo se hace una propuesta didáctica que concibe el desarrollo de la actividad procedimental a través de las representaciones semióticas de los procesos de variación y cambio, como contexto para la formación de conceptos en el proceso de enseñanza aprendizaje del Cálculo Diferencial, y que utiliza el asistente matemático DERIVE, como escenario para su desarrollo. El marco teórico está dado por la utilización de múltiples registros de representaciones semióticas como vía para lograr el nexo entre la comprensión conceptual y procedimental. Se demostró, a través de la validación experimental, que con este enfoque los estudiantes mejoran, de forma significativa, su desempeño en la solución de ejercicios matemáticos, lo cual constituyó el objetivo de la investigación.

Palabras clave: variación y cambio, cálculo diferencial, propuesta didáctica

\footnotetext{
${ }^{1}$ Artículo de investigación científica

${ }^{2}$ Universidad Autónoma de Santo Domingo, Recinto Santiago de los Caballeros, República Dominicana, Departamento de Matemática, Máster en Matemática Aplicada, estudiante de doctorado en Ciencias Pedagógicas E-mail: abaez@isa.edu.do

${ }^{3}$ Universidad de Camagüey Ignacio Agramonte Loynaz, Doctora en Ciencias Pedagógicas, Presidenta del Comité Latinoamericano de Matemática Educativa, Premio Nacional de la Sociedad Cubana de Matemática y Computación. E-mail: olga.perez@reduc.edu.cu

${ }^{4}$ Universidad de Camagüey Ignacio Agramonte Loynaz, Máster en Administración, Profesor principal de año académico, Facultad de Ciencias Aplicadas a la Industria. E-mail: bartolo.triana@reduc.edu.cu
} 


\title{
Didactic proposal based on multiple forms of semiotic representation of mathematical objects to develop the teaching learning process of differential calculus
}

\begin{abstract}
In the article a didactic proposal is made, which envisions the development of the procedural activity through the semiotic representations of the processes of variation and change, as a scenario for the formation of concepts in the learning process of the Differential Calculus, and how it uses the mathematical assistant DERIVE, as a platform for its performance. The theoretical framework is given by the use of multiple registers of semiotic representations as a way to achieve the nexus between conceptual and procedural understanding. It was demonstrated, through the experimental validation, that with this approach the students significantly improve their performance in the solution of mathematical exercises, which was the objective of the research.
\end{abstract}

Keywords: variation and change, differential calculus, didactic proposal

Proposta didática baseada em múltiplas formas de representação semiótica de os objetos matemáticos para desenvolver o processo de ensino-aprendizagem do cálculo diferencial.

\section{Resumo}

No artigo faz-se uma proposta didática que concebe o desenvolvimento da atividade procedimental através das representações semióticas dos processos de variação e câmbio, como contexto para a formação de conceitos no processo de ensino aprendizagem do Cálculo Diferencial, e que utiliza o assistente matemático DERIVE, como cenário para o seu desenvolvimento. O marco teórico está dado pela utilização de múltiplos registros de representações semióticas como via para alcançar o nexo entre a compreensão conceitual e de procedimentos. Pôde-se demonstrar, através da validação experimental, que com esta abordagem os estudantes melhoram significativamente o seu desempenho na solução de exercícios matemáticos, fator que constituiu o objetivo da pesquisa.

Palavras-chaves: variação e mudança, cálculo diferencial, proposta didática. 


\section{Introducción}

En el proceso enseñanza-aprendizaje del Cálculo Diferencial se manifiestan insuficiencias en su tratamiento didáctico, al hacerse un marcado énfasis en la solución de tareas con un enfoque algorítmico a través de habilidades algebraicas (Gordillo \& Pino-Fan, 2016; Caicedo \& Díaz, 2011), lo que, generalmente limita la comprensión significativa de los conceptos y métodos de pensamiento matemático.

En este sentido, Rittle-Johnson, Siegler \& Alibali (2001) sugieren un tratamiento didáctico que interconecte la comprensión conceptual y la habilidad procesal en matemática, para combinar la acción con la comprensión, y que los estudiantes puedan saber por qué se ejecutan los procedimientos que requieren la solución de problemas; así como poder conjugar la capacidad de saber qué se hace y por qué se hace.

Una de las vías para lograr el nexo entre lo conceptual y lo procedimental es los procesos de tratamiento y conversión de las múltiples formas de representación semiótica de los objetos matemáticos, lo que se ha dominado como registros de representación semiótica (Duval, 1993).

Según este autor, lo anterior requiere del desarrollo de tareas donde se deba trabajar la formación de una representación, que pueda identificarse como perteneciente a un registro dado; además del proceso y transformación de una representación dentro del registro donde fue creado, y finalmente la conversión, es decir, la transformación de una representación semiótica de un registro a otro.

El objetivo de la investigación estuvo orientado a mejorar el desempeño de los estudiantes en la solución de ejercicios matemáticos, a través de la implementación de una propuesta didáctica que concibe el desarrollo de la actividad procedimental a través de las representaciones semióticas de los procesos de variación y cambio, como contexto para la formación de conceptos en el proceso de enseñanza aprendizaje del Cálculo Diferencial, y que utiliza el asistente matemático DERIVE, como escenario para el tratamiento y conversión de las múltiples formas de representación semiótica de los objetos matemáticos.

\section{Antecedentes}

Desde la década de los años 80 y hasta la actualidad, han tomado auge diversas investigaciones orientadas a la formación y desarrollo del pensamiento variacional como elemento fundamental para la enseñanza y aprendizaje del Cálculo Diferencial, con la intención de propiciar un aprendizaje con significado para los estudiantes, sobre los procesos de variación y cambio, y con sentido en el ámbito de su futura área profesional, lo que se ha ido convirtiendo en una línea de investigación muy importante en la Didáctica del Cálculo Diferencial (Ruiz, 2009; Salinas, 2016; Pulgarín, 2016).

Sin embargo, aún persisten insuficiencias didácticas, pues, según Silva \& Rico (2016), la enseñanza del Cálculo Diferencial, tiende a centrarse en una práctica algorítmica y algebraica, evaluando los conocimientos adquiridos desde lo algorítmico, convirtiéndose este fenómeno en un círculo vicioso para poder tener niveles aceptables de éxito, pero sin lograr una comprensión significativa de los conceptos y métodos de pensamiento variacional.

Es por eso que se hace necesario perfeccionar el trabajo didáctico, desde lo conceptual y lo procedimental, tratando de combinar la acción con la comprensión, de modo que los estudiantes sepan por qué se ejecutan los procedimientos que se requieren para la solución de problemas matemáticos; conjugando la capacidad de saber qué se hace y por qué se hace (Zúñiga, 2007; Prada-Núñez, Hernández-Suárez \& Ramírez-Leal, 2016).

En relación a esta problemática, Dolores (2013), plantea que, en el discurso escolar de la 
matemática, no se presta la adecuada atención a la formación de las ideas variacionales tan necesarias para la comprensión y uso de los conceptos del Cálculo Diferencial, lo que conlleva a que muchos estudiantes tengan dificultades en el aprendizaje de esta asignatura, predominando las prácticas algorítmicas y algebraicas.

Además, los métodos y procedimientos empleados para abordar los procesos de variación y el cambio están estructurados, atendiendo en lo fundamental al estudio de la razón de cambio y su tratamiento algebraico $\mathrm{y}$ en pocas ocasiones a procedimientos en variaciones geométricas, limitándose el desempeño de los estudiantes en la interpretación, planteamiento y resolución de problemas (Chávez, García, González, Quezada, Álvarez, Quiñones y Sandoval, 2016).

\section{Marco teórico}

Para el logro del objetivo de la investigación se asume como referente teórico la existencia de múltiples formas de representación semiótica de los conceptos matemáticos de Duval (1999), las cuales denominó como registros de representación semiótica.

Según este autor la utilización de múltiples registros de representaciones semióticas y la transferencia entre estos registros es una vía ideal para lograr el nexo entre la comprensión conceptual y la procedimental, y que cuando solo se trabajan estos desde un solo modo de representación se limita el desempeño de los estudiantes en la solución de tareas matemáticas.

Como los conceptos matemáticos no son directamente accesibles a la percepción, es de obligada referencia la noción de representación, y para lograr la comprensión de los conceptos es necesario desarrollar tareas donde se deba trabajar tres tipos de acciones con los registros de representación semiótica: la primera orientada a la formación de una representación identificándola como perteneciente a un registro dado; la segunda orientada al proceso y transformación de una representación dentro del registro donde fue creado (acción de tratamiento), y finalmente la transformación de una representación semiótica de un registro a otro (acción de conversión).

En las acciones de tratamiento y conversión, los autores de la investigación acuden al razonamiento inductivo - deductivo para favorecer el proceso de argumentación, utilizandoy evaluando estrategias que permitan reconocer las características invariantes de fenómenos que representen situaciones de variación y cambio en diferentes registros de representación semiótica.

El trabajo didáctico con el uso de las representaciones semióticas constituye un medio de comunicación; para que los estudiantes puedan asignarle significados a los conceptos matemáticos, dentro de un sistema semiótico, aunque sea rudimentario, describiendo cómo funcionan ciertos sistemas de representación y efectuando tratamientos (operaciones, cálculos y modelación).

\section{El derive como escenario para la implementación de la propuesta}

En la actualidad el uso de las tecnologías para la enseñanza en la educación superior se hace cada vez más indispensable, en este sentido Beltrán (2011), destaca a la educación virtual como elemento esencial del desarrollo educativo en los ambientes universitarios y argumenta la necesidad de su uso para el desarrollo de propuestas didácticas.

En relación al proceso de enseñanza aprendizaje de la Matemática en la educación superior, son variadas las propuestas, desde diferentes perspectivas, pero que de forma general develan la necesidad y actualidad de considerar el uso de la tecnología para la implementación de propuestas didácticas que pretendan mejorar el desempeño de los estudiantes, a través de la comprensión conceptual procedimental. 
En relación a lo anterior Taborda y Meneses (2015), proponen el uso de los laboratorios virtuales como estrategia didáctica para desarrollar el pensamiento lógico de los estudiantes, así como Gutiérrez, Ariza y Mujica (2014), que describen cuáles deben ser las estrategias didácticas para el uso de herramientas virtuales en el proceso de enseñanza aprendizaje de la Matemática.

Teniendo en cuenta estos aspectos, en la investigación se asume la utilización de la tecnología como escenario para implementar la propuesta didáctica, seleccionando al software matemáticos DERIVE por ser un asistente matemático que, según Porres, Pecharromán y Ortega (2017), permite coordinar diferentes registros de representación del concepto sin excesivo formalismo, ahorra tiempo y facilita el desarrollo del pensamiento lógico, permite el desarrollo de la argumentación reflexiva, el trabajo desde la inducción-deducción, la motivación y una fundamentación más sólida de las construcciones mentales.

El DERIVE es utilizado en las acciones de tratamiento y conversión de los conceptos matemáticos y en el razonamiento inductivo y deductivo de las tareas que incluye la propuesta didáctica, aprovechando las posibilidades que brinda este asistente matemático para resolver las actividades previstas.

Para evitar obstáculos relacionados con el uso del DERIVE, al inicio del semestre se desarrollan clases preparatorias para que los estudiantes sepan utilizarlo y conozcan los comandos e instrucciones que se requieren para su utilización, concibiéndolo como un recurso tan imprescindible como el libro de texto.

\section{Metodología}

La propuesta didáctica estuvo conformada por dos fases de trabajo orientadas a la comprensión de los conceptos involucrados en los procesos de variación y cambio, a través del razonamiento inductivo-deductivo desde la actividad procedimental, con el objetivo de que los estudiantes mejoraran su desempeño en la solución de ejercicios en el proceso de enseñanza aprendizaje del Cálculo Diferencial.

Para el logro de lo anterior se plantearon tareas en las que se tuvieran que desarrollar y argumentar los procedimientos de conversión de un objeto en diversos registros de representación semiótica, lo que requirió de la realización de acciones sobre las diversas representaciones, de forma tal que se favoreciera la comprensión de los conceptos del Cálculo Diferencial, siendo estas acciones las que caracterizaron la actividad procedimental en el registro semiótico del concepto representado.

Para llevar lo anterior a la práctica se orientó el trabajo en dos fases que tuvieron una estrecha relación de coordinación entre ellas:

- Fase 1: Orientación inductiva en las acciones de conversión de representación semiótica de los procesos de variación y cambio.

- Fase 2: Orientación deductiva en la justificación de los procesos de variación $\mathrm{y}$ cambio a partir de sus representaciones semióticas.

La fase 1 estuvo dirigida a la preparación de los estudiantes para que, a través de la argumentación reflexiva del funcionamiento de la conversión de las representaciones semióticas de los procesos de variación y cambio, pudieran establecer conclusiones generales sobre los conceptos estudiados.

Esto requirió del docente orientar adecuadamente a los estudiantes para que reconocieran la importancia de la formación y tratamiento de los registros semióticos objetos de estudio, su naturaleza o funcionalidad de cada rasgo esencial, así como las reglas que rigen el tratamiento del registro. 
Para lograr lo anterior, se plantearon tareas donde se hizo uso de distintas representaciones, enfatizando que con la progresiva articulación entre ellas, se enriquecía el significado y el conocimiento de los conceptos estudiados, así como considerar que en la medida en que los estudiantes conocieran y supieran tratar con distintos tipos de representaciones, podrían realizar un análisis más profundo del proceso de variación y cambio y disponer de un mayor número de herramientas matemáticas para resolver las tareas asignadas.

En este caso se plantearon tareas con variadas representaciones semióticas, a través de las cuales se manipularon los conceptos objeto de estudio, se plantearon hipótesis y se trazaron las pautas para el trabajo con el tratamiento y la conversión de los diferentes registros de representación semiótica de los procesos de variación y cambio.

La fase 2 estuvo dirigida a preparar a los estudiantes para que dadas las características generales de las diferentes representaciones de los procesos de variación y cambio pudieran hacer un razonamiento lógico de las características particulares de los conceptos estudiados.

Para concretar lo anterior se trabajó en el análisis de las diferentes representaciones de los procesos de variación y cambio, estableciendo las relaciones lógicas entre las unidades que lo constituyen, realizando una argumentación en forma precisa de sus reflexiones con respecto a sus interpretaciones y así llegar a conclusiones particulares del concepto objeto de estudio.

En esta fase se solicitó a los estudiantes la justificación de las representaciones semióticas de los procesos de variación y cambio, a través de argumentos deductivos, utilizando analogías y conjeturas, con un debate reflexivo sobre la validez de diferentes proposiciones vinculadas a los fenómenos de variación y cambio, para que así pudieran detectar las inconsistencias en los razonamientos propios y ajenos, por medio de actividades cognitivas de tratamiento y conversión de las representaciones semióticas.

Como hilo conductor de las dos fases que caracterizan la propuesta didáctica, se concibió la práctica de la argumentación reflexiva para favorecer el nexo entre la comprensión conceptual y procedimental, a través de los diferentes registros de representación semiótica de los conceptos estudiados.

\section{Implementación de la propuesta}

Las tareas se implementaron como trabajos investigativos orientados a generar la argumentación reflexiva, a través de tareas matemáticas extra que reflejaran procesos de variación y cambio en el contexto físico o ingenieril, dirigidas a la mejora del desempeño de los estudiantes en la solución de ejercicios en el proceso de enseñanza aprendizaje del Cálculo Diferencial.

Cada tarea estuvo compuesta por tres etapas:

1. Presentación y análisis de la tarea, gestión de la información que ella ofrece a través de la identificación y comprensión de patrones variacionales que representa.

2. Realización de la actividad procedimental, utilizando el software matemático DERIVE, representando los procesos de variación y cambio identificados, en los diferentes registros de representación semiótica, reflexionando $\mathrm{y}$ argumentando sobre el funcionamiento de la conversión de las representaciones, estableciendo conclusiones generales sobre los conceptos estudiados.

3. Análisis de las características generales de las diferentes representaciones de los procesos de variación y cambio para hacer un razonamiento lógico de las características particulares de los conceptos estudiados, utilizando el software matemático DERIVE. 


\section{Entrega del informe desarrollado por cada equipo de trabajo}

Análisis grupal, reflexivo-valorativo, del trabajo realizado para debatir sobre el por qué se ejecutaron los procedimientos que requirió la solución de problemas; y para reflexionar sobre la necesidad de conjugar la capacidad de saber qué se hace y por qué se hace para poder comprender los conceptos estudiados.

Para ejemplificar la propuesta, y por limitaciones del espacio disponible, se ejemplificarán solamente la etapa 1 y 2 de la implementación de la propuesta a través del siguiente problema.

Dos automóviles salen de la misma intersección de calles perpendiculares, uno de norte a sur a $40 \mathrm{~km} / \mathrm{h}$ y otro de este a oeste a $30 \mathrm{~km} / \mathrm{h}$. Si ambos se desplazan sin detenerse, cuando el primero ha recorrido un $\mathrm{km}$, ¿cuán rápido se incrementa la distancia entre ellos? Para la gestión de la información que esta tarea ofrece, en relación a los patrones variacionales que ella representa, se sugirieron las siguientes interrogantes: ¿Cuál es la variable independiente en el problema?, ¿Cuál es la dependencia de variables que el problema revela?, ¿Existen otras variables dependientes en el problema?, ¿Cuáles son las relaciones entre las variables?, ¿Cómo se manifiestan las relaciones de variación y cambio entre las variables del problema? y ¿Qué se necesita encontrar para resolver el problema?

Para la realización de la actividad procedimental, utilizando el software matemático DERIVE, se representaron los procesos de variación y cambio identificados en los diferentes registros de representación semiótica, como se muestra en la figura 1.
Figura 1 Materialización semiótica del problema en los registros gráficos y analíticos

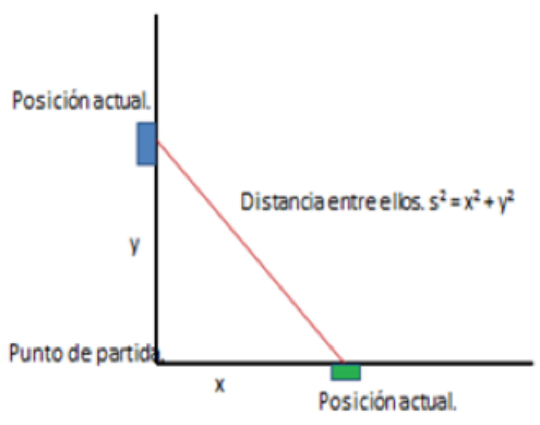

En este caso se reflexionó y argumentó cómo fue posible obtener el registro analítico, y se valoraron las diferentes opciones de obtener el registro analítico, a través de las nociones previas que tenían sobre el cálculo de distancia entre dos puntos, y a través de la interpretación geométrica utilizando el teorema de Pitágoras.

Para poder llegar a conclusiones sobre el análisis del problema estudiado se analizó que si los dos automóviles se han desplazado el mismo tiempo, entonces, $\frac{y}{40}=\frac{x}{30}$, o bien: $\frac{3}{4} y=x$, por lo que el proceso de variación y cambio puede ser representado de la siguiente forma $2 \frac{d s}{d t}=2 x \frac{d x}{d t}+2 y \frac{d y}{d t}, \mathrm{y}$ teniendo en cuenta que $\frac{3}{4} y=x$ se tiene entonces que $\frac{3}{4} \frac{d y}{d t}=\frac{d x}{d t}$.

Se reflexionó y argumentó sobre las relaciones entre los elementos expuestos en la gráfica, y en la ecuación encontrada, distinguiendo los elementos que distinguen a la variación y el cambio, enfatizándose en el aspecto conceptual de los procesos de variación y cambio, en el registro de representación algebraico y geométrico, incentivando la significación del concepto de derivada, no sólo desde lo geométrico y lo algebraico; sino, a través de la comprensión de los procesos de variación y cambio, a través de su funcionalidad en el problema dado.

Se hizo un debate sobre las propiedades y reglas, relacionadas con el concepto derivada, siguiendo argumentos deductivos informales y exigiendo el uso del lenguaje matemático para argumentar la solución del problema. 


\section{Validación experimental de los resultados}

La propuesta se implementó en las carreras de Ingeniería de la Universidad Autónoma de Santo Domingo, Recinto Santiago de los Caballeros, República Dominicana, en los cursos 2012-2013, 2013-2014, 2014$2015,2015-2016$, en condiciones naturales y siguiendo la metodología desarrollada por Triana-Hernández (2014).

Para el desarrollo de la investigación se planteó la siguiente hipótesis: Si se implementa una propuesta didáctica, que concibe el desarrollo de la actividad procedimental a través de las representaciones semióticas de los procesos de variación y cambio, como contexto para la formación de conceptos en el proceso de enseñanza aprendizaje del Cálculo Diferencial, y que utiliza el asistente matemático DERIVE, como escenario para el tratamiento y conversión de las múltiples formas de representación semiótica de los objetos matemáticos, entonces se contribuirá a que los estudiantes mejoren el desempeño en la solución de ejercicios en el proceso de enseñanza aprendizaje del Cálculo Diferencial.

Se realizó una validación mediante el criterio de expertos, utilizando el método DELPHI, con el objetivo de determinar los indicadores para poder determinar la variable independiente: desempeño de los estudiantes en la solución de ejercicios matemáticos.

Como resultado de este estudio se precisaron los siguientes indicadores para valorar la variable independiente:

- Los estudiantes identifican y manipulan relaciones, patrones, transformaciones, procesos algebraicos y geométricos.

- Los estudiantes reconocen la existencia y el significado de la variación en un fenómeno modelado matemáticamente.
- Los estudiantes distinguen

- Los estudiantes establecen relaciones entre los diferentes elementos que distinguen la variación, relacionan propiedades y reglas siguiendo argumentos deductivos informales.

- Los estudiantes comunican y reflexionan matemáticamente $\mathrm{y}$ usan el lenguaje matemático para argumentar la solución de problemas.

- Los estudiantes manipulan relaciones, transformaciones, procesos algebraicos y geométricos, en relación al problema dado.

En el estudio experimental la variable independiente fue manipulada por los investigadores, y se caracterizó por ser fue longitudinal y prospectivo. El grupo de control se estudia en los cursos académicos 2012-2013, 2013-2014, y grupo experimental en 2014-2015, 2015-2016.

Todos los estudiantes matriculados en dichos cursos formaron parte de la muestra de investigación, analizándose las calificaciones obtenidas al terminar el curso de Cálculo Diferencial, las cuales fueron clasificadas como excelente (5), bien (4), regular (3) y mal (2).

Para el análisis de los resultados se utilizó el software estadístico R, y se aplicó la prueba de $\square 2$, realizándose todas las comparaciones posibles entre los cursos experimentales y de control(2012-2013 con 2014-2015, 2012-2013 con 2015-2016, 2013-2014 con 2014-2015 y 2013-2014 con 2015-2016), posteriormente se hizo una última comparación entre los dos grupos experimentales (2014-2015 con 2015-2016 para ver si existieron diferencias significativas entre los resultados obtenidos en ambos grupos.

Se pudo comprobar que entre los dos grupos experimentales no hubo diferencia 
significativa y que hubo mejores resultados en los grupos experimentales con respecto a los grupos donde no se realizó el experimento. Además, el análisis porcentual de los indicadores determinados por los expertos, para poder valorar el desempeño de los estudiantes en la solución de ejercicios en el proceso de enseñanza aprendizaje del Cálculo Diferencial, arrojó como resultado que hubo mejoría significativa en el desempeño en relación al desarrollo del pensamiento variacional, ya que, de forma general, logran examinar la existencia y el significado de la variación en un fenómeno modelado matemáticamente, distinguiendo los elementos en la variación.

Se logró que dada una situación variacional, representada por un fenómeno matemático extra, logren modelarlo matemáticamente, y lo caractericen por los elementos que distinguen a la variación, estableciendo relaciones entre dichos elementos, a través de tablas, gráficas $\mathrm{y}$ otras formas de representación.

\section{Conclusiones}

La investigación sugiere un enfoque diferente para desarrollar el proceso de enseñanza aprendizaje del Cálculo Diferencial, articulando la comprensión conceptual y la habilidad procesal, en el estudio de procesos de variación y cambio, a través del tratamiento y conversión de las múltiples formas de representación semiótica de los objetos matemáticos.

Se demostró a través de la validación experimental, que con este enfoque los estudiantes mejoran, de forma significativa, su desempeño en la solución de ejercicios en el proceso de enseñanza aprendizaje del Cálculo Diferencial, identificando $\mathrm{y}$ manipulando patrones de variación $\mathrm{y}$ cambio en contextos analíticos, algebraicos y numéricos, reconociendo la existencia de procesos de variación y cambio, así como su significado y elementos esenciales, para de esta forma comprender los conceptos matemáticos asociados a estos procesos.

Se logró, además, que los estudiantes manipularan relaciones, transformaciones, procesos algebraicos y geométricos, en relación a los problemas dados, argumentando su solución.

El uso de la tecnología como escenario para desarrollar la propuesta didáctica, fue esencial para motivar a los estudiantes, y para desarrollar las fases previstas en la propuesta didáctica, y aunque se realizó con el software matemático DERIVE, es posible la utilización de cualquier otro software que permita el trabajo de tratamiento y conversión de las múltiples formas de representación semiótica de los objetos matemáticos.

Aunque los resultados fueron satisfactorios, hubo dificultades por la no correspondencia entre el enfoque de las tareas del libro de texto y las planteadas en la propuesta, así como las dificultades con la utilización del DERIVE por parte de los estudiantes.

\section{Agradecimientos}

Agradecimiento al apoyo brindado a la Universidad Autónoma de Santo Domingo, Recinto Santiago de los Caballeros, República Dominicana y al Proyecto Nacional del Departamento de Matemática de la Universidad de Camagüey, "Perfeccionamiento de la enseñanza de la Matemática" (código PP221LH053), asociado al Programa Nacional Problemas Actuales del Sistema Educativo Cubano. Perspectivas de desarrollo, del Ministerio de Educación de Cuba. 


\section{Referencias}

Beltrán, J. (2011). La educación virtual en Colombia: la implementación de las TIC en la educación superior. Academia y Virtualidad, $4(1), 6-21$.

Caicedo, S., \& Díaz, L. (2011). Pensamiento variacional y sentencias e igualdades numéricas aditivas. Revista UNIMAR (58), 98-105.

Chávez, O., García, S., González, J., Quezada, M., Álvarez, M., Quiñones, M., \& Sandoval, O. (2016). Uso de tecnología para la diferenciación a través del concepto de variación. Revista de Orientación Educacional, 83(94), 30-57.

Dolores, C. (2013). La variación y la derivada. México: Ediciones Díaz Santos.

Duval, R. (1999). Semiosis y pensamiento humano: registros semióticos y aprendizajes intelectuales (Vol. 1). (M. Vega, Ed.) Cali, Colombia: Universidad del Valle.

Duval, R. (1993). Registros de representación semiótica y funcionamiento cognitivo del pensamiento. México: Grupo Editorial Iberoamérica.

Gordillo, W., \& Pino-Fan, L. (2016). Una Propuesta de Reconstrucción del Significado Holístico de la Antiderivada. Bolema, 30(55), 535.

https://doi.org/10.1590/1980-4415v30n55a12

Gutiérrez, L., Ariza, L., \& Mujica, J. (2014). Estrategias didácticas en el uso y aplicación de herramientas virtuales para el mejoramiento en la enseñanza del cálculo integral. Revista academia y virtualidad, 7(2), 64-75.

https://doi.org/10.18359/ravi.319

Porres, M., Pecharromán, C., \& Ortega, T. (2017). Aportaciones de DERIVE y del cálculo y del cálculo mental al aprendizaje de la integral definida. PNA, 11(2), 125-153.

Prada-Núñez, R., Hernández-Suárez, C.A. \& Ramírez-Leal, P. (2016). Comprensión de la noción de función y la articulación de los registros semióticos que la representan entre estudiantes que ingresan a un programa de Ingeniería. Revista Científica, 2(25).

https://doi.org/10.14483//udistrital.jour. RC.2016.25.a3

Pulgarín, J. (2016). Generalización de patrones geométricos: proyecto de aula para desarrollar pensamiento variacional en estudiantes de 9-12 a-os. Tesis Doctoral, Universidad Nacional de Colombia-Sede Medellín.

Rittle-Johnson, B., Siegler, R., \& Alibali, M. (2001). Developing conceptual understanding and procedural skill in mathematics: An iterative process. Journal of educational psychology, 93(2), 346.

https://doi.org/10.1037/0022-0663.93.2.346

Ruiz, E. (2009). Diseño de estrategias de enseñanza para el concepto de variación en áreas de ingeniería. Innovación Educativa, 9(46), 27-39.

Salinas, C. (2016). Laboratorio de pensamiento variacional: Una experiencia para estudiantes de poblaciones vulnerables. Revista Internacional de Aprendizaje en Ciencia, Matemáticas y Tecnología, 3(2), 93-102.

Silva, M., \& Rico, S. (2016). ¿Por qué los estudiantes no asisten a las horas de consulta de Cálculo I? Revista Colombiana de Matemática Educativa, 1(1b), 80.

Taborda, S., \& Meneses, E. (2015). Laboratorio virtual de matemáticas como estrategia didáctica para fomentar el pensamiento lógico. Academia y Virtualidad, 8(2), 73-84.

https://doi.org/10.18359/ravi.1424

Triana-Hernández, B. (2014). La disciplina Química para la formación ambiental del 
ingeniero agrónomo. Revista Cubana de Química, 26(3), 259-275.

Zúñiga, L. (2007). El cálculo en carreras de ingeniería: un estudio cognitivo. Revista latinoamericana de investigación en matemática educativa, 10(1), 145-175. 